\title{
EXACT EQUATION FOR WILSON LOOPS IN 2-DIMENTIONAL EUCLIDEAN SPACE
}

\author{
MOFAZZAL AZAM \\ THEORETICAL PHYSICS DIVISION, CENTRAL COMPLEX, BHABHA ATOMIC \\ RESEARCH CENTRE \\ TROMBAY, MUMBAI-400085, INDIA
}

\begin{abstract}
We derive an exact equation for simple self non-intersecting Wilson loops in non-abelian gauge theories with gauge fields interacting with fermions in 2-dimensional Euclidean space.
\end{abstract}


In the context of quantum gravity, string theory and black holes, t'Hooft [1] and Susskind [2] have proposed the holographic principle. According to this principle, it may be possible to describe the world in terms of variables living on a two dimensional surface much like information coded on a two dimensional hologram. On the hologram, Susskind [3] has argued that the non-local objects such as Wilson loops are the basic observables.These observables have come to be known as Precursors.This, indeed, makes Wilson loops very important objects of study.Long ago, it was shown by this author that in the set of all posssible Wilson loops, there exists a smaller set which generates the whole set of observables [4]. It was also shown that, when the potential and field strenghth are analytic, there exist gauge invariant local generators for the Wilson loops [5]. This last result, as pointed out by Giddings and Lippert [6], may possibly be relevant while connecting local events deep inside 3 -space with Wilson loops on the hologram.

There are many other important works associated with the Wilson loops.Notable among them are the works of Polyakov [7], Migdal and Makeenko [8], Jeviki and Sakita [9] .Polyakov poined out that at the classical level the equations of motion of the Wilson loops in pure gauge theory are very much like classical equations of motion for chiral fields.In the quantum theory, Migdal and Makeenko have derived equations of motion for Wilson loops in a pure gauge theory in the large $\mathrm{N}$ limit .On the other hand, Jeviki and Sakita have tried to formulate the quantum theory in terms of Wilson loops by making a change of variable in the functional integral for gauge fields.Recently, Agarwal and Rajeev have provided a geometrically unified picture of all the three above mentioned approaches [10].

As mentioned above, the importance of precursors makes us look at all these and any other results connected with Wilson loops very seriously.In this paper, we will be concerned with Wilson loops in a non-abelian gauge theory with gauge fields interacting with fermions in 2-dimensional Euclidean space.In fact, we will derive an exact, closed equation for simple self non-intersecting Wilson loops.

It turns out that 2-dimensional ( or rather $1+1$ dimensional) gauge theories have been fertile ground for many new and important ideas. The most significant result is Schwinger's 
demonstration of mass term for photons in $1+1$ dimensional QED [11]. For $\mathrm{SU}(N)$ gauge theories t'Hooft has provided a complete solution in the large $N$ limit [12]. There has also been extensive study of this model by Belevedre et al [13]. Another interesting result is by the present author and Naik [14. In this publication it was shown that the order parameter of glueball condensation and discrete chiral symmetry are related, in other words, glueball condesation would lead to the breaking of discrete chiral symmetry.

The present work is motivated by an attempt to obtain results similar to Schwinger's in 2-dimensional QED.(From now on, we will consider only 2-dimensional Euclidean space).We obtain, in the non-abelian gauge theory a closed equation for smeared gauge invariant field srtength. However, the equation is non-local and we can not interpret it as an equation for massive pseudoscalar field.In the case of abelian $U(1)$ gauge theory, this equation exactly reproduces Schwinger's result.To see what we mean, let us rederive Schwinger's result in a very simple and straight forward manner.The equation of motion is given by,

$$
\partial^{\mu} F_{\mu \nu}(x)=g J_{\nu}(x)
$$

where $F_{\mu \nu}$ is the field strength and $J_{\nu}(x)=\bar{\psi}(x) \gamma_{\nu} \psi(x), \bar{\psi}(x), \psi(x)$ are the Dirac spinors and $\gamma_{\nu}$ are the Dirac gamma matrices in two Euclidean dimensions. The axial vector current $J_{\mu}^{5}(x)=\bar{\psi}(x) \gamma_{\mu} \gamma^{5} \psi(x)$, has an anomaly given by [15,16,

$$
\partial^{\mu} J_{\mu}^{5}(x)=\frac{g}{\pi} \epsilon^{\mu \nu} F_{\mu \nu}(x)
$$

where $\epsilon^{\mu \nu}=-\epsilon^{\nu \mu}$ is antisymmetric tensor in 2-dimensional space. Note that in 2-dimensional space,$F_{\mu \nu}(x)=\epsilon^{\mu \nu} \phi(x)$, where $\phi(x)$ is a pseudo-scalar field.In terms of the pseudo-scalar field $\phi(x)$, the equation of motion and the anomaly equation becomes,

$$
\begin{aligned}
& \partial_{\mu} \phi(x)=g J_{\mu}^{5}(x) \\
& \partial_{\mu} J_{\mu}^{5}(x)=\frac{g}{\pi} \phi(x)
\end{aligned}
$$

From the equations above we easily obtain,

$$
\partial^{\mu} \partial_{\mu} \phi(x)=g \partial^{\mu} J_{\mu}^{5}(x)=\frac{g^{2}}{\pi} \phi(x)
$$


This can also be written as,

$$
\square \phi(x)-\frac{g^{2}}{\pi} \phi(x)=0
$$

By analytic continuation of the time co-ordinate to Minkowskii space, we obtain the equation for a pseudo-scalar particle of mass $m=\frac{g}{\sqrt{\pi}}$. This was the result obtained by Schwinger for two dimensional QED.In a non-abelian gauge theory with gauge fields interacting with fermions, we will try to derive an equation analogous to Eq.(5), which will turn to be a nonlocal equation for the area derivative of the Wilson loop.This area derivative of the Wilson loop is, in fact, the smeared gauge invariant field strength.

First of all, let us introduce a few definitions.Let $S U(N)$ be the non-abelian gauge group with generators $T_{a}$.

$$
\left[T_{a}, T_{b}\right]=f_{a b}^{c} T_{c}
$$

$f_{a b}^{c}$ are the structure constants of the Lie algebra of the group $S U(N)$. The potential and the field strength $A_{\mu}(x)$ and $F_{\mu \nu}(x)$ are,

$$
A_{\mu}(x)=A_{\mu}^{a}(x) T_{a} \quad ; \quad F_{\mu \nu}(x)=F_{\mu \nu}^{a}(x) T_{a}
$$

Let $C_{x y}$ be a curve starting at $x$ and ending at $y . w\left(C_{x y}\right)$ is defined as,

$$
w\left(C_{x y}\right)=P \exp \left(\int_{C_{x y}} A_{\mu} d x^{\mu}\right)
$$

If $C_{x x}$ is a curve starting at $x$ and also ending at $x$, then,

$$
w\left(C_{x x}\right)=P \exp \left(\oint_{C_{x x}} A_{\mu} d x^{\mu}\right)
$$

Note that under gauge transformation, $w\left(C_{x x}\right)$ transforms exactly like the field strength $F_{\mu \nu}(x)$. The Wilson loop is given by,

$$
w(C)=\operatorname{Tr}\left(P \exp \left(\oint_{C} A_{\mu} d x^{\mu}\right)\right)
$$

The area derivative of the Wilson loop at the point $x$, is given by [7, [8] 


$$
\frac{\delta w(C)}{\delta \sigma_{\mu \nu}(x)}=\operatorname{Tr}\left[F_{\mu \nu}(x) w\left(C_{x x}\right)\right]
$$

In two dimensional space,

$$
F_{\mu \nu}=\epsilon_{\mu \nu} \Phi=\epsilon_{\mu \nu} \Phi^{a} T_{a} ; \sigma_{\mu \nu}=\epsilon_{\mu \nu} \sigma
$$

where $\epsilon_{\mu \nu}=-\epsilon_{\nu \mu}$ is the antisymmetric tensor in 2-dimensional space. This allows us to write the area derivative of the Wilson loop as,

$$
\frac{\delta w(C)}{\delta \sigma(x)}=\operatorname{Tr}\left[\Phi(x) w\left(C_{x x}\right)\right]
$$

The area derivative can be looked upon as smeared gauge invariant field strength. Note that for abelian gauge fields,

$$
\frac{\delta w(C)}{\delta \sigma_{\mu \nu}(x)}=F_{\mu \nu}(x) w\left(C_{x x}\right)=w\left(C_{x x}\right) F_{\mu \nu}(x)
$$

The non-local derivative $\partial_{\mu}^{x}$ is defined as [7,8],

(1) For a local function $f(x)$,

$$
\partial_{\mu}^{x} f(x)=\partial_{\mu} f(x)
$$

(2) For non-local objects such as $w\left(C_{x y}\right)$,

$$
\partial_{\mu}^{x} w\left(C_{x y}\right)=-A_{\mu}(x) w\left(C_{x y}\right) ; \quad \partial_{\mu}^{y} w\left(C_{x y}\right)=w\left(C_{x y}\right) A_{\mu}(y)
$$

(3) For non-local objects $w\left(C_{x x}\right)$, corresponding to closed loops $C_{x x}$,

$$
\partial_{\mu}^{x} w\left(C_{x x}\right)=\left[w\left(C_{x x}\right), A_{\mu}(x)\right]
$$

Note that for abelian gauge theories,

$$
\partial_{\mu}^{x} w\left(C_{x x}\right)=\left[w\left(C_{x x}\right), A_{\mu}(x)\right]=0
$$

In 2-dimensional non-abelian gauge theory, the equation of motion is given by,

$$
D^{\mu} F_{\mu \nu}=g J_{\nu}
$$


which can also be written as,

$$
D_{\mu} \Phi=g \epsilon_{\mu \nu} J^{\nu}=g J_{\mu}^{5}
$$

The axial vector current $J_{\mu}^{5}$ has an anomaly given by [13],

$$
D^{\mu} J_{\mu}^{5 a}=\frac{g}{2 \pi} \Phi^{a}
$$

Taking non-local derivative $\partial_{\mu}^{x}$ of both side of Eq.(13), after some algebraic manipulation, we obtain

$$
\partial_{\mu}^{x} \frac{\delta w(C)}{\delta \sigma(x)}=\operatorname{Tr}\left[D_{\mu} \Phi(x) w\left(C_{x x}\right)\right]
$$

Using the equation of motion, we obatin

$$
\partial_{\mu}^{x} \frac{\delta w(C)}{\delta \sigma(x)}=\operatorname{Tr}\left[D_{\mu} \Phi(x) w\left(C_{x x}\right)\right]=g \operatorname{Tr}\left[J_{\mu}^{5} w\left(C_{x x}\right)\right]
$$

Applying again the derivative $\partial_{\mu}^{x}$ to both side of Eq.(23) and using the anomaly equation, Eq.(21) we get

$$
\partial^{\mu x} \partial_{\mu}^{x} \frac{\delta w(C)}{\delta \sigma(x)}=g \operatorname{Tr}\left[D^{\mu} J_{\mu}^{5} w\left(C_{x x}\right)\right]=\frac{g^{2}}{2 \pi} \operatorname{Tr}\left[\Phi(x) w\left(C_{x x}\right)\right]
$$

Let $\square^{x} \equiv \partial^{\mu x} \partial_{\mu}^{x}$.Then using Eq.(13), we can write the above equation Eq.(24) as,

$$
\square^{x} \frac{\delta w(C)}{\delta \sigma(x)}=\frac{g^{2}}{2 \pi} \frac{\delta w(C)}{\delta \sigma(x)}
$$

This is the basic equation.It is non-local and is valid for any size of the loop. This is, in fact, an equation for the smeared gauge invariant field strength. In abelian $U(1)$ gauge theory, using the anomaly equation Eq.(2), we obtain

$$
\square^{x} \frac{\delta w(C)}{\delta \sigma(x)} \equiv \partial^{\mu x} \partial_{\mu}^{x} \frac{\delta w(C)}{\delta \sigma(x)}=\frac{g^{2}}{\pi} \frac{\delta w(C)}{\delta \sigma(x)}
$$

The equation above for abelian gauge theory should be supplemented by equation, Eq.(18) , i.e.,

$$
\partial_{\mu}^{x} w\left(C_{x x}\right)=0
$$


Note that, in the non-abelian gauge theory there is a local equation for the field strength.The equation is given by,

$$
D^{\mu} D_{\mu} \Phi(x)=\frac{g}{2 \pi} \Phi(x)
$$

The non-local equation Eq.(25), for the smeared gauge invariant field strength is, in fact, obtained by multiplying both sides of the equation, Eq.(28) by $w\left(C_{x x}\right)$, taking trace and using the definitions of area derivative and the non-local derivative. 


\section{REFERENCES}

[1] G.'t Hooft, gr-qc/9310026

[2] L.Susskind, J.Math. Phys.36, 6377(1995),hep-th/9409089

[3] L.Susskind and N.Toumbas, hep-th/9909013

[4] M.Azam, Phys. Rev. D 35, 2043(1987); ibid 43, 4148(1991)

[5] M.Azam, Phys. Rev. D 40, 3541(1989)

[6] S.B.Giddings and M.Lippert, Phys. Rev. D 65 024006(2002)

[7] A.M.Polyakov, Nucl.Phys.B164 171(1980)

[8] Yu.M.Makeenko and A.A.Migdal, Phys.Lett B88 269 (1979); Nucl.Phys.B188 269 (1981)

[9] A.Jeviki and B.Sakita, Nucl.Phys.B165 511 (1980); ibid B185 89 (1981)

[10] A.Agarwal and S.G.Rajeev, hep-th/0202095

[11] J.Schwinger, Phys. Rev. 1282425 (1962)

[12] G.'t Hooft, Nucl.Phys. B75 461(1975)

[13] L.V.Belvedere, J.A.Swieca, K.A.Rothe and B.Schroer, Nucl.Phys. B153 112 (1979)

[14] M.Azam and S.Naik, Mod.Phys.Lett. A4 2549(1989)

[15] K.A.Johnson, Phys. Letters 5 253(1963)

[16] S.Coleman, Commun.Math.Phys. 31 259(1973) 